\title{
Circ_0078607 inhibits the progression of ovarian cancer via regulating the miR-32-5p/ SIK1 network
}

Yangqiu Jin ${ }^{1 *}$ and Hui Wang ${ }^{2}$

\begin{abstract}
Background: Circular RNA (circRNA) has been shown to be involved in the regulation of human disease progression, including ovarian cancer (OC). Circ_0078607 was found to participate in OC progression. But its function and mechanism in OC deserve further exploration.

Methods: The expression levels of circ_0078607, salt-inducible kinase 1 (SIK1) and microRNA (miR)-32-5p were examined by qRT-PCR. And the protein expression levels of SIK1, metastasis marker and apoptosis marker were determined using western blot analysis. EDU staining, colony formation assay, transwell assay and flow cytometry were used to detect the proliferation, migration, invasion and apoptosis of cells. Moreover, dual-luciferase reporter assay was employed to verify the interaction between miR-32-5p and circ_0078607 or SIK1. Xenograft models were constructed to perform in vivo experiments.
\end{abstract}

Results: Circ_0078607 and SIK1 were downregulated in OC tissues and cells. Overexpressed circ_0078607 and SIK1 could inhibit OC cell proliferation, migration, invasion, and promote apoptosis. MiR-32-5p could be sponged by circ_0078607, and its overexpression could reverse the suppressive effect of circ_0078607 on OC progression. Furthermore, SIK1 was a target of miR-32-5p, and circ_0078607 could regulate SIK1 by sponging miR-32-5p. The inhibitory effect of circ_0078607 on OC progression also could be reversed by SIK1 silencing. In vivo experiments showed that circ_0078607 reduced OC tumorigenesis by regulating the miR-32-5p/SIK1 axis.

Conclusion: Circ_0078607 could serve as a sponge of miR-32-5p to regulate SIK1 expression, thereby inhibiting OC progression.

\section{Highlights}

1. Circ_0078607 acts as a sponge of miR-32-5p.

2. MiR-32-5p targets SIK1.

Keywords: Ovarian cancer, circ_0078607, miR-32-5p, SIK1

\footnotetext{
*Correspondence: adt11md@163.com

1 Department of Reproductive Medicine, Liaocheng People's Hospital,

NO.67, Dongchang West Road, Liaocheng City 252000, Shandong Province, China

Full list of author information is available at the end of the article
}

\section{Introduction}

Ovarian cancer $(\mathrm{OC})$ is a malignant tumor that occurs in the ovary and is the biggest disease that seriously threatens women's health $[9,20]$. Since most patients are in the advanced stage at the time of diagnosis, the treatment of permits use, sharing, adaptation, distribution and reproduction in any medium or format, as long as you give appropriate credit to the original author(s) and the source, provide a link to the Creative Commons licence, and indicate if changes were made. The images or other third party material in this article are included in the article's Creative Commons licence, unless indicated otherwise in a credit line to the material. If material is not included in the article's Creative Commons licence and your intended use is not permitted by statutory regulation or exceeds the permitted use, you will need to obtain permission directly from the copyright holder. To view a copy of this licence, visit http://creativecommons.org/licenses/by/4.0/. The Creative Commons Public Domain Dedication waiver (http://creativeco mmons.org/publicdomain/zero/1.0/) applies to the data made available in this article, unless otherwise stated in a credit line to the data. 
OC is more difficult and the mortality of patients is high $[12,17]$. Although the current treatment technology of OC continues to improve, the prognosis of most patients is still not ideal $[4,8]$. Therefore, elucidating the molecular mechanisms affecting the occurrence of OC may provide a theoretical basis for finding effective therapeutic targets of OC.

As a special non-coding RNA, circular RNA (circRNA) has received a lot of attention in recent years. Studies have found that circRNA is widely and diversely present in a variety of biological cells and has the regulating effect on gene expression $[1,2]$. Importantly, the researchers have found that the expression of circRNA is closely related to the progression of many human diseases, including OC $[19,22]$. For example, circPLEKHM3 had been discovered to inhibit OC cell growth and metastasis [28]. On the contrary, circWHSC1 was upregulated in $\mathrm{OC}$ and was found to promote the proliferation, migration and invasion of OC [30]. Circ_0078607 is a newly discovered circRNA in recent years. Zhang et al. proposed that circ_0078607 was lowly expressed in OC and it might act as tumor suppressor to hinder OC progression [29]. However, there are still few studies on circ_0078607, and its role and mechanism in OC deserve further investigation.

Salt-inducible kinase 1 (SIK1) is a serine/threonine protein kinase, belongs to SIK family [24]. SIK1 is confirmed to have important function in regulating cell biological function and maintaining homeostasis $[18,26]$. With the development of research, more and more studies have confirmed that SIK1 expression is also associated with the progression of many cancers, including hepatocellular carcinoma [15], pancreatic cancer [16] and colorectal cancer [7]. It had been reported that SIK1 knockdown could promote the proliferation of OC [3]. Therefore, SIK1 might play an anti-cancer role in OC.

CircRNA has been confirmed to act as a sponge of microRNA (miRNA), and then release miRNA's inhibitory effect on mRNA to indirectly regulate mRNA expression [5, 13]. In our research, we found a significant negative correlation between circ_0078607 and SIK1. We speculated that there might be a miRNA that could interact with circ_0078607 and SIK1. Therefore, we conducted this research around the hypothesis of the circRNA/miRNA/mRNA axis.

\section{Materials and methods}

\section{Tissue samples}

A total of 43 patients diagnosed with $\mathrm{OC}$ were recruited from Liaocheng People's Hospital. OC tumor tissues $(n=43)$ and adjacent normal tissues $(n=43)$ of all patients were collected after surgery. Informed consent was obtained from each patient. The study was approved by the Ethics Committee of Liaocheng People's Hospital. The clinicopathologic features of OC patients were shown in Table 1.

\section{Cell culture and transfection}

Human OC cell lines (HEY and ES-2) and normal epithelial ovarian cell line (IOSE80) were obtained from Biovector NTCC (Beijing, China). All cells were cultured in RPMI-1640 medium (Gibco, Grand Island, NY, USA) supplemented with 10\% FBS (Gibco) and 1\% double antibiotics (Gibco) at $37^{\circ} \mathrm{C}$ in an incubator with $5 \% \mathrm{CO}_{2}$. For cell transfection, HEY and ES-2 cells were seeded into 12-well plates. The circ_0078607 overexpression vector (oe-circ_0078607) and its control (NC), the pcDNA overexpression vector and small interference RNA (siRNA) of SIK1 (SIK1 and si-SIK1) or their controls (pc-DNA and si-NC), miR-32-5p mimic (miR-32-5p) and its control (miR-NC), as well as the lentivirus overexpression vector of circ_0078607 (lenti-circ_0078607) and its control (lenti-NC) were constructed by GenePharma (Shanghai, China) and were transfected into cells by Lipofectamine 3000 Reagent (Invitrogen, Carlsbad, CA, USA).

\section{qRT-PCR}

The RNA were extracted by TRIzol reagent (Invitrogen). The cDNA was obtained using SuperScript IV First-Strand Synthesis System (Invitrogen). Basing on the specific primers, qRT-PCR was performed by SYBR Green (Solarbio, Beijing, China). Relative expression

Table 1 The correlation between circ_0078607 expression and clinicopathologic features of ovarian cancer patients

\begin{tabular}{|c|c|c|c|c|}
\hline \multirow[t]{2}{*}{ Characteristics } & \multirow[t]{2}{*}{$n=43$} & \multicolumn{2}{|c|}{ Circ_0078607 expression } & \multirow[t]{2}{*}{$P$ value } \\
\hline & & Low $(n=21)$ & High $(n=22)$ & \\
\hline Age (years) & & & & 0.455 \\
\hline$<55$ & 18 & 10 & 8 & \\
\hline$\geq 55$ & 25 & 11 & 14 & \\
\hline Histological type & & & & 0.083 \\
\hline I & 14 & 10 & 4 & \\
\hline$\|-\| \|$ & 29 & 11 & 18 & \\
\hline FIGO stage & & & & $0.010^{*}$ \\
\hline$|-| \mid$ & 20 & 14 & 6 & \\
\hline III & 23 & 7 & 16 & \\
\hline $\begin{array}{l}\text { Lymph node metas- } \\
\text { tasis }\end{array}$ & & & & $0.021^{*}$ \\
\hline No & 23 & 15 & 8 & \\
\hline Yes & 20 & 6 & 14 & \\
\hline CA125 & & & & 0.273 \\
\hline$<35$ & 13 & 8 & 5 & \\
\hline$\geq 35$ & 30 & 13 & 17 & \\
\hline
\end{tabular}

FIGO the International Federation of Gynecology and Obstetrics; ${ }^{*} P<0.05$ 
was examined using $2^{-\Delta \Delta C T}$ method and normalized by GAPDH or U6. The sequences of primers were shown as follows: circ_0078607, F 5'-AGATCCTGAGACGCATTG CT-3', R 5'-AGCCTAAGGTGAATGCTCCA-3'; SIK1, F 5'-CTCCGGGTGGGTTTTTACGAC-3', R 5'-CTGCGT TTTGGTGACTCGATG-3'; miR-32-5p, F 5'-GCCGAG TATTGCACATTACTAA-3', R $5^{\prime}$-GTGCAGGGTCCG AGGT-3'; GAPDH, F 5'-CTCTGCTCCTCCTGTTCG AC-3', R 5'-CGACCAAATCCGTTGACTCC-3'; U6, F 5'-CTCGCTTCGGCAGCACATA-3', R 5'-CGAATT TGCGTGTCATCCT-3'.

\section{Western blot (WB) analysis}

RIPA buffer (Solarbio) was utilized for extracting total protein, and then the protein was quantified by BCA Kit (Sangon, Shanghai, China). Next, the proteins were separated by SDS-PAGE gel and electro-blotted to PVDF membrane (Beyotime, Shanghai, China). The membrane was incubated with the primary antibodies against SIK1 (51045-1-AP, 1:1000), E-cadherin (20874-1-AP, 1:5000), N-cadherin (22018-1-AP, 1:2000), matrix metalloproteinase 9 (MMP9, 10375-2-AP, 1:1000), Bcl2-associated x (Bax, 50599-2-Ig, 1:5000) or GAPDH (10494-1-AP, 1:20,000). After further incubating with Goat Anti-Rabbit IgG (SA00001-2, 1:10,000), the protein bands in the membranes were visualized using Ultrasensitive ECL Dection Kit (Proteintech, Rosemont, IL, USA). All antibodies were obtained from Proteintech.

\section{EDU staining}

Click-iT ${ }^{\circledR}$ EDU Imaging Kit was obtained from Invitrogen. According to the kit instructions, the EDU positive cells were measured to evaluate cell proliferation.

\section{Colony formation assay}

After transfection for $48 \mathrm{~h}, \mathrm{HEY}$ and ES-2 cells were collected and then inoculated into 6-well plates. After culturing for 2 weeks, the cells were fixed with $4 \%$ paraformaldehyde and stained with crystal violet. Afterwards, the number of colonies was counted under a microscope (Leica, Wetzlar, Germany).

\section{Transwell assay}

The transwell chambers (24-well, $8 \mu \mathrm{m}$ ) (Corning Inc., Corning, NY, USA) was used to measure cell migration and invasion. An additional Matrigel (Corning Inc.) was needed to pre-coat the upper chamber for detecting cell invasion. Briefly, HEY and ES-2 cells suspended in serum-free medium were placed in upper chambers. In addition, the complete medium was added into the lower chambers. $24 \mathrm{~h}$ later, the cells transferred to the lower chamber were fixed and stained, and its numbers were counted under a microscope $(100 \times)$.

\section{Flow cytometry}

Cell apoptosis was measured using the Annexin V-FITC/PI Apoptosis Detection Kit (BestBio, Shanghai, China). In brief, HEY and ES-2 cells were harvested after transfection for $48 \mathrm{~h}$. The cells were then suspended with Binding Buffer, followed by staining with Annexin V-FITC and PI in the dark. Cell apoptosis rate was analyzed by flow cytometry and counted using the CellQuest software.

\section{Dual-luciferase reporter assay}

The pGL3 luciferase reporter vectors for the wide-type (WT) and mutate-type (MUT) of circ_0078607 (WT/ MUT-circ_0078607) or SIK1 3'UTR (WT/MUT-SIK13'UTR) were synthesized by GenePharma. The reporter vectors were co-transfected into HEY and ES-2 cells with miR-NC or miR-32-5p mimic. $48 \mathrm{~h}$ later, relative luciferase activity was detected using the Dual-Lucy Assay Kit (Solarbio).

\section{Xenograft models}

The animal experiment was supported by the Animal Ethics Committee of Liaocheng People's Hospital. Ten female BALB/c nude mice (Vital River, Beijing, China) were divided into 2 groups ( $n=5$ per group). HEY cells $\left(5 \times 10^{6}\right.$ cells $)$ transfected with lenti-NC or lenticirc_0078607 were subcutaneously injected into the back of the nude mice. The tumor length and width were measured every 7 days to calculated tumor volume. After 28 days, the mice were euthanized and the tumor was collected. In addition, paraffin sections were prepared from tumor tissue to perform Ki-67 immunohistochemical (IHC) staining using Ki-67 Kit (Sangon).

\section{Statistical analysis}

All experiment was performed in triplicate, and all independent experiments were set for 3 times to take the average value. All data were represented as mean \pm standard deviation. Statistical analysis was performed using GraphPad Prism 7.0. Student's $t$-test and one-way ANOVA followed by Tukey's post-hoc test were used to analyze the differences. Pearson correlation analysis was used for analyzing the correlation among circ_0078607, miR-32-5p and SIK1. $P<0.05$ was considered significant.

\section{Results}

Circ_0078607 and SIK1 were lowly expressed and positively correlated in $\mathrm{OC}$ tissues

In OC tumor tissues, we discovered that circ_0078607 was downregulated compared with that in adjacent normal tissues (Fig. 1A). Then, we divided the 43 
A

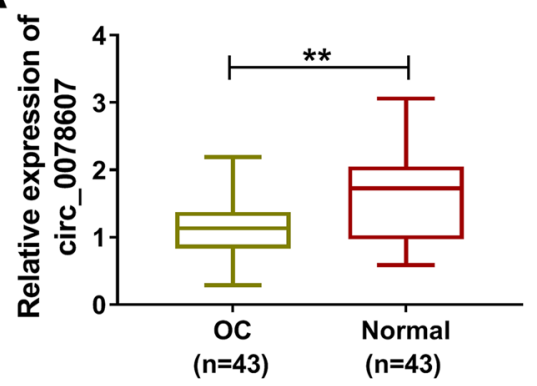

C

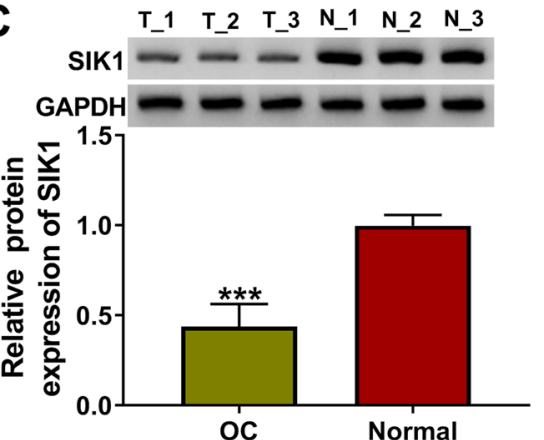

B

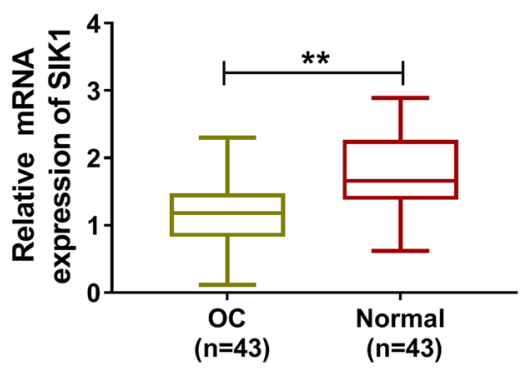

D

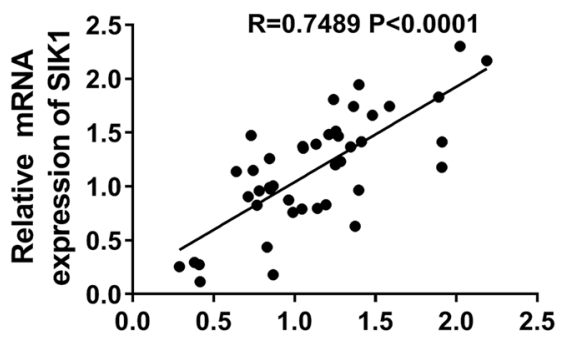

Relative expression of circ_0078607

Fig. 1 Circ_0078607 and SIK1 were lowly expressed and positively correlated in OC tissues. A The expression of circ_0078607 in OC tumor tissues $(\mathrm{OC}, n=43$ ) and adjacent normal tissues (Normal, $n=43$ ) was detected by qRT-PCR. B-C The mRNA and protein expression levels of SIK1 in OC $(n=3)$ and Normal $(n=3)$ were measured by qRT-PCR and WB analysis. D Pearson correlation analysis was used to assess the correlation between circ_0078607 and SIK1 in OC tumor tissues $(n=43)$. A-C, Student's t-test. ${ }^{* *} P<0.01$, ${ }^{* * *} P<0.001$

OC patients into low and high circ_0078607 expression groups according to the median expression level of circ_0078607. Through analyzing the correlation between circ_0078607 expression and clinicopathologic features of $\mathrm{OC}$ patients, we discovered that circ_0078607 expression was correlated with the FIGO stage and lymph node metastasis of OC patients (Table 1). Besides, SIK1 expression also was lower in OC tumor tissues than that in adjacent normal tissues at the mRNA level and protein level (Fig. 1B-C). Surprisingly, the correlation analysis results showed that there was a significant positive correlation between the expression of circ_0078607 and SIK1 in OC tumor tissues (Fig. 1D). These results suggested that circ_0078607 and SIK1 might play the important roles in the progression of $\mathrm{OC}$.

\section{The expression of circ_0078607 and SIK1 was decreased in OC cells}

At the same time, we measured circ_0078607 and SIK1 expression on OC cells. The results showed that compared to the IOSE80 cells, circ_0078607 expression and SIK1 mRNA and protein expression were markedly lowly expressed in OC cells (HEY and ES-2) (Fig. 2AC). To confirm the regulation of circ_0078607 on SIK1, we constructed the circ_0078607 overexpression vector.
After transfecting with oe-circ_0078607 into HEY and ES-2 cells, circ_0078607 expression was significantly enhanced (Fig. 2D). The detection results of SIK1 mRNA and protein expression suggested that circ_0078607 overexpression could remarkably promote SIK1 expression in HEY and ES-2 cells (Fig. 2E-F). Our data showed that circ_0078607 positively regulated SIK1 in OC cells.

\section{Overexpressed circ_0078607 suppressed proliferation, migration, invasion and promoted apoptosis in OC cells} Then, we assessed the biological functions of HEY and ES-2 cells after overexpressing circ_0078607. EDU staining and colony formation assay results suggested that circ_0078607 overexpression could inhibit the EDU positive cells and reduce the number of cloned HEY and ES-2 cells (Fig. 3A-B), showing that the proliferation of OC cells could be repressed by circ_0078607 overexpression. Besides, the numbers of migrated and invaded HEY and ES-2 cells also were obviously inhibited by circ_0078607 overexpression (Fig. 3C-D). The results of flow cytometry indicated that overexpressed circ_0078607 could enhance the apoptosis rate of HEY and ES-2 cells (Fig. 3E). In addition, the protein levels of $\mathrm{N}$-cadherin and MMP9 were markedly decreased, while the protein levels of E-cadherin and Bax were notably increased in HEY and ES-2 cells overexpressed circ_0078607 (Fig. 3F). These 


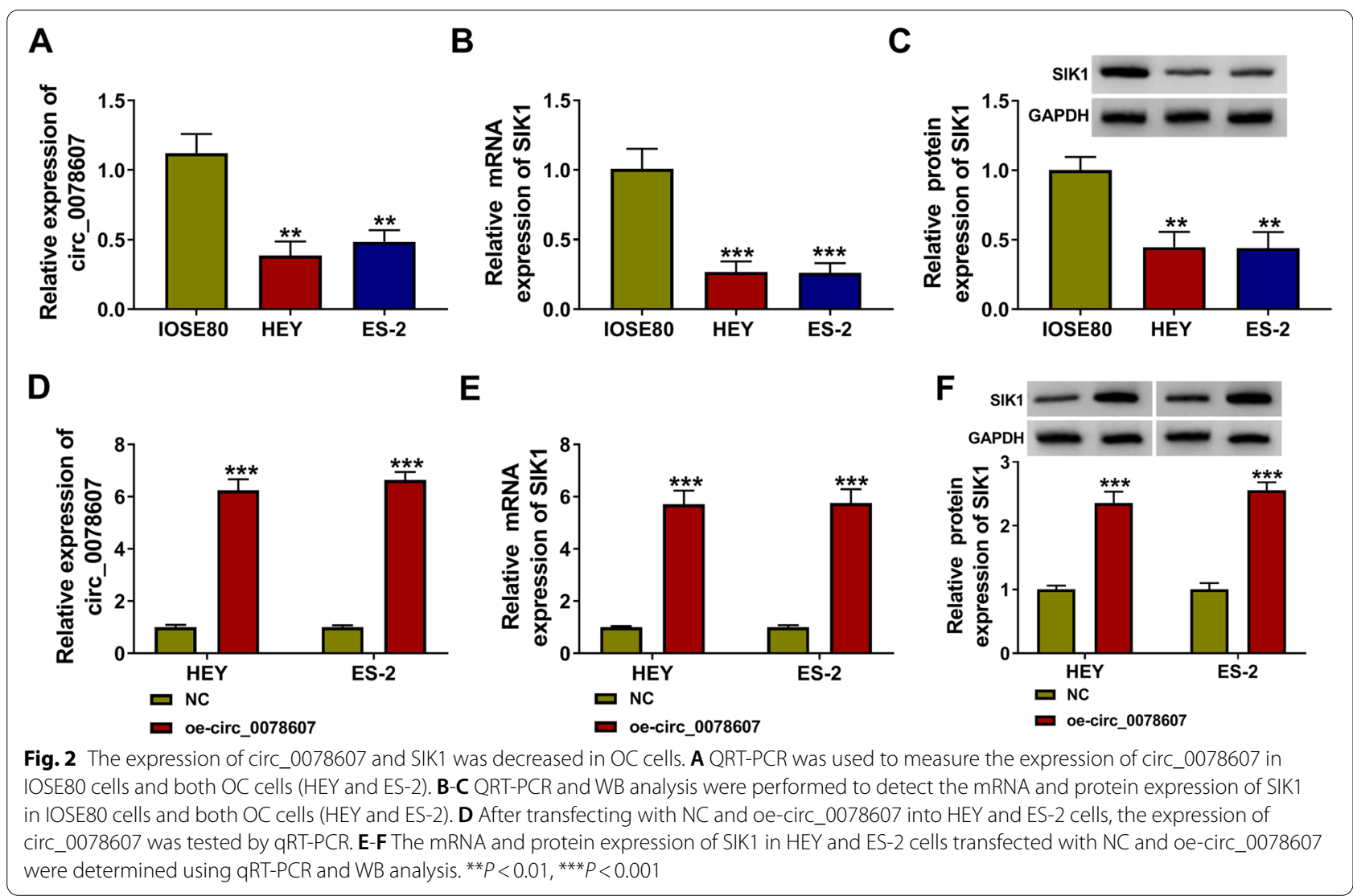

data indicated that circ_0078607 might play a negative role in $\mathrm{OC}$ progression.

\section{SIK1 overexpression inhibited OC progression}

To explore the role of SIK1 in OC progression, we also built the pc-DNA overexpression vector of SIK1. By detecting SIK1 mRNA and protein expression after transfection, we confirmed that pc-DNA SIK1 overexpression vector indeed increased SIK1 expression (Fig. 4A-B), indicating the successful transfection. Subsequently, we evaluated the effect of SIK1 overexpression on OC cell progression. Functional experiments showed that overexpressed SIK1 could significantly inhibit EDU positive cells, the number of colonies and the numbers of migrated and invaded cells in HEY and ES-2 cells (Fig. 4C-F). Also, SIK1 overexpression promoted the apoptosis rate, inhibited N-cadherin and MMP9 protein levels, while accelerated E-cadherin and Bax protein levels in HEY and ES-2 cells (Fig. 4G-H). Therefore, our data revealed that SIK1 might hinder OC progression, which was similar to the role of circ_0078607 in OC.

\section{Circ_0078607 could act as a sponge of miR-32-5p}

To explore the targeted miRNA of circ_0078607, we used the starbase and circbank software to perform bioinformatic prediction, and a total of 9 candidate miRNAs were screened out (Fig. 5A). After overexpression of circ_0078607, we found that miR-32-5p expression was the most obvious inhibited (Fig. 5B), so miR-32-5p was selected as the target miRNA of circ_0078607 for this study. According to the binding sites, the WT/MUT-circ_0078607 vectors were generated (Fig. 5C). To carry out follow-up experiments, we constructed miR-32-5p mimic and confirmed that it could indeed promote miR-32-5p expression in HEY and ES-2 cells (Fig. 5D). Besides, dual-luciferase reporter assay was used to verify the interaction between miR-32-5p and circ_0078607, and the results showed that miR-32-5p overexpression could obviously reduce the luciferase activity of WT-circ_0078607 vector without affecting that of the MUT-circ_0078607 vector (Fig. 5E). In OC tumor tissues and cells, we discovered that miR-32-5p expression was remarkably increased compared with that in the adjacent normal tissues and IOSE80 cells, respectively (Fig. 5F-G). And miR-32-5p expression could be obviously inhibited by circ_0078607 overexpression in HEY and ES-2 cells (Fig. 5H). Through the Pearson correlation analysis, we found that there had a negative correlation between miR-32-5p and circ_0078607 in OC tumor tissues 


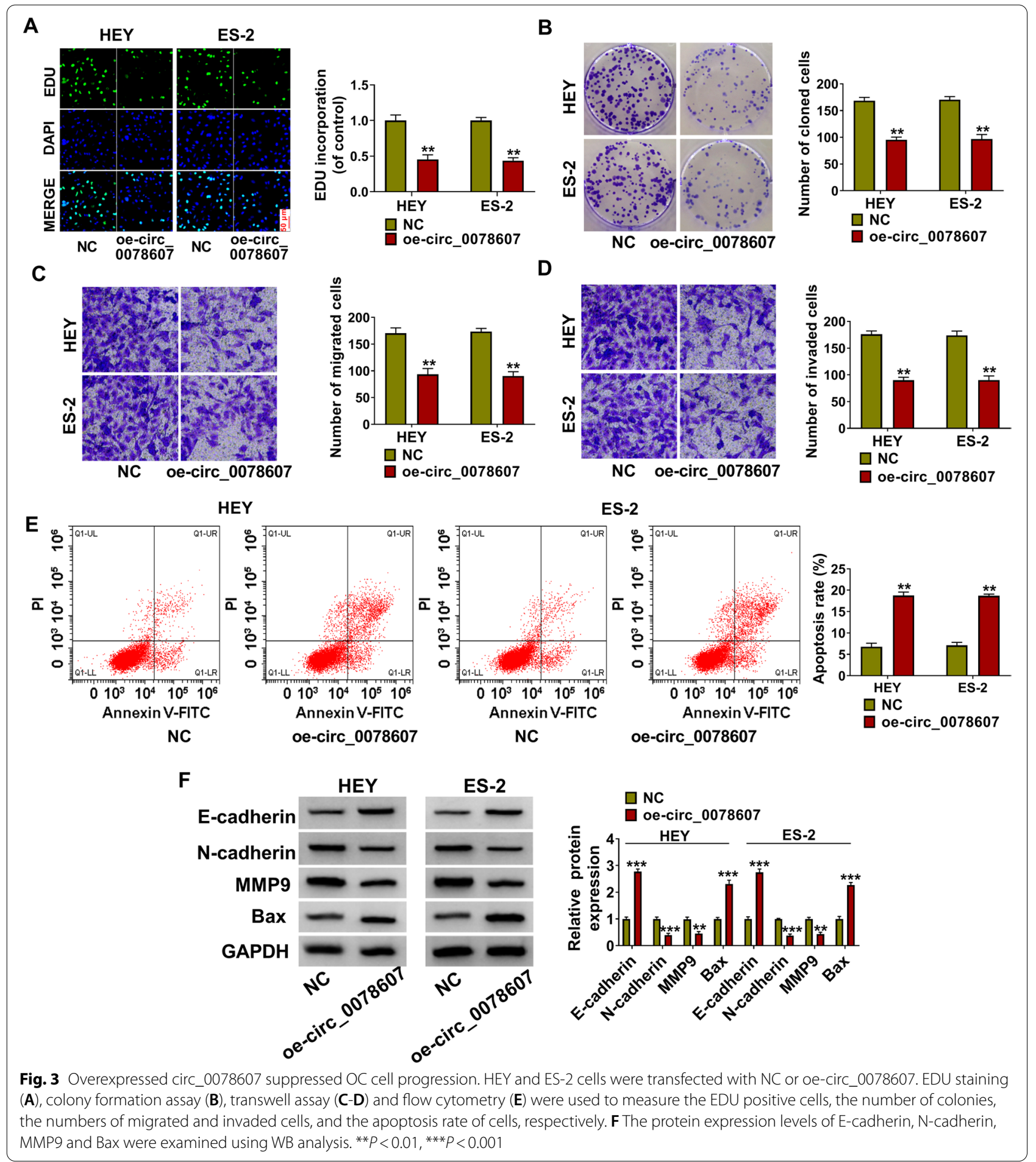

(Fig. 5I). These results illuminated that miR-32-5p was a targeted miRNA of circ_0078607.
MiR-32-5p reversed the regulation of circ_0078607 on OC proliferation, metastasis and apoptosis

To further confirm that circ_0078607 regulated OC progression via sponging miR-32-5p, oe-circ_0078607 and miR-32-5p mimic were co-transfected into HEY and ES-2 


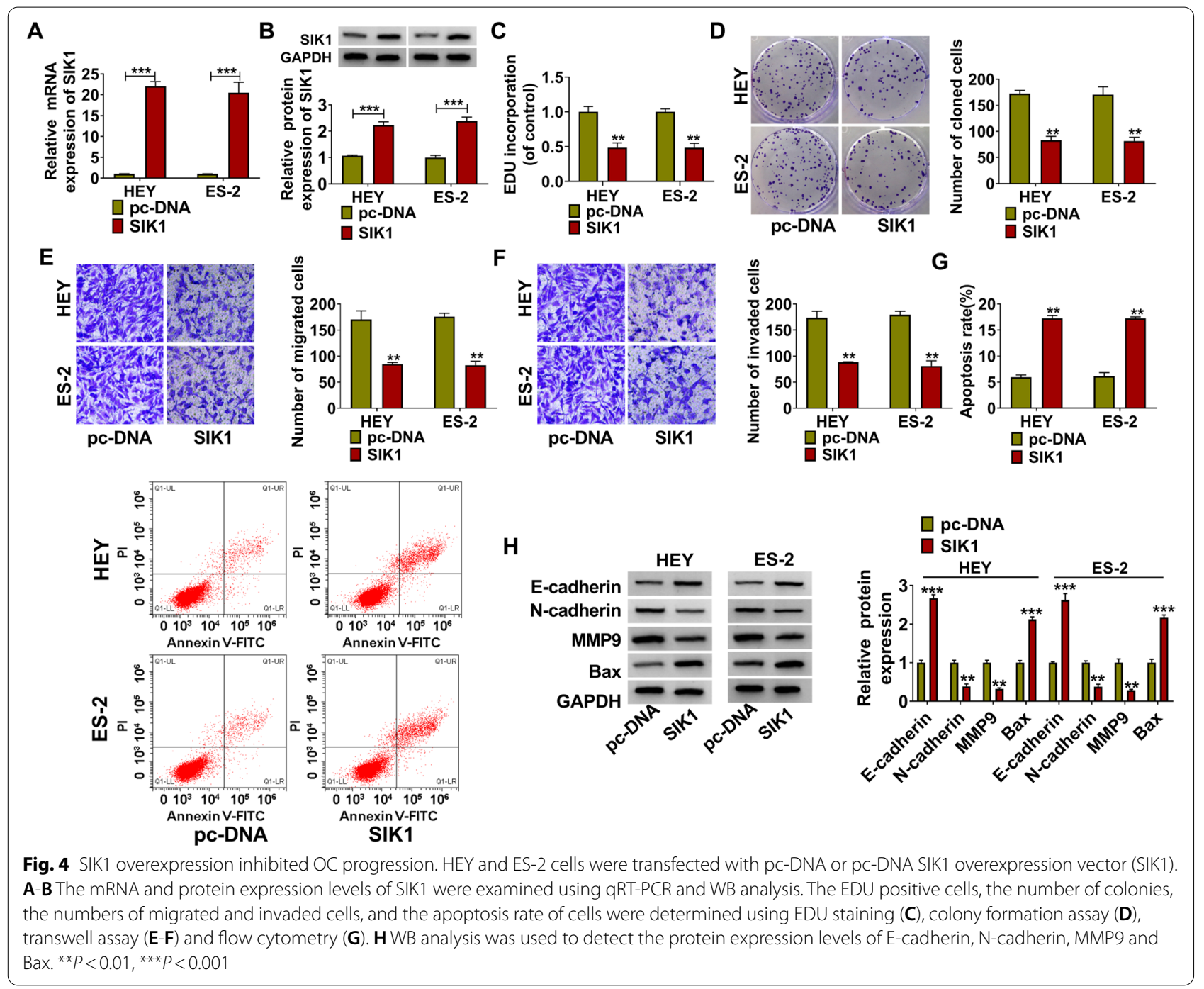

cells. Through detecting miR-32-5p, we confirmed that the addition of miR-32-5p mimic could promote miR32-5p expression inhibited by circ_0078607 overexpression (Fig. 6A). EDU staining, colony formation assay and transwell assay results showed that the suppressive effect of circ_0078607 overexpression on EDU positive cells, the number of colonies and the numbers of migrated and invaded cells could be reversed by miR-32-5p overexpression (Fig. 6B-E and Supplementary Fig. 1A-C). Furthermore, the apoptosis rate of HEY and ES-2 cells promoted by circ_0078607 also could be abolished by the addition of miR-32-5p mimic (Fig. 6F and Supplementary Fig. 1D). The decreasing effect of circ_0078607 on N-cadherin and MMP9 protein levels, as well as the increasing effect on E-cadherin and Bax protein levels also were recovered by overexpressing miR-32-5p (Fig. 6G). All data revealed that miR-32-5p was involved in the regulation of circ_0078607 on OC progression.

\section{SIK1 was a target of miR-32-5p}

Using several online databases, we selected 8 genes that have been reported to play tumor suppressor function in OC as candidate genes. QRT-PCR was used to detect the influence of miR-32-5p overexpression on the mRNA expression of these genes, and it was found that miR32-5p overexpression had the most significant inhibition on SIK1 expression (Fig. 7A). Therefore, SIK1 was used as the target of miR-32-5p in this study. The sequences of WT/MUT-SIK1-3'UTR vector were shown in Fig. 7B. The results of dual-luciferase reporter assay suggested that the luciferase activity of WT-SIK1-3'UTR vector could be inhibited by miR-32-5p mimic, while that of the MUT-SIK1-3'UTR vector was not affected by miR32-5p mimic (Fig. 7C). Moreover, we found that the mRNA and protein expression levels of SIK1 could be reduced by miR-32-5p overexpression (Fig. 7D-E). In OC tumor tissues, SIK1 mRNA expression was discovered 


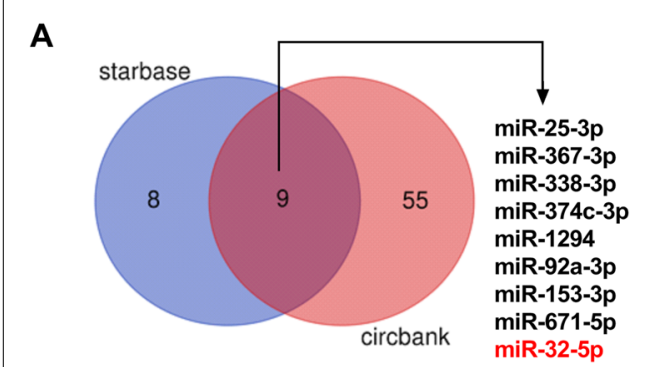

C
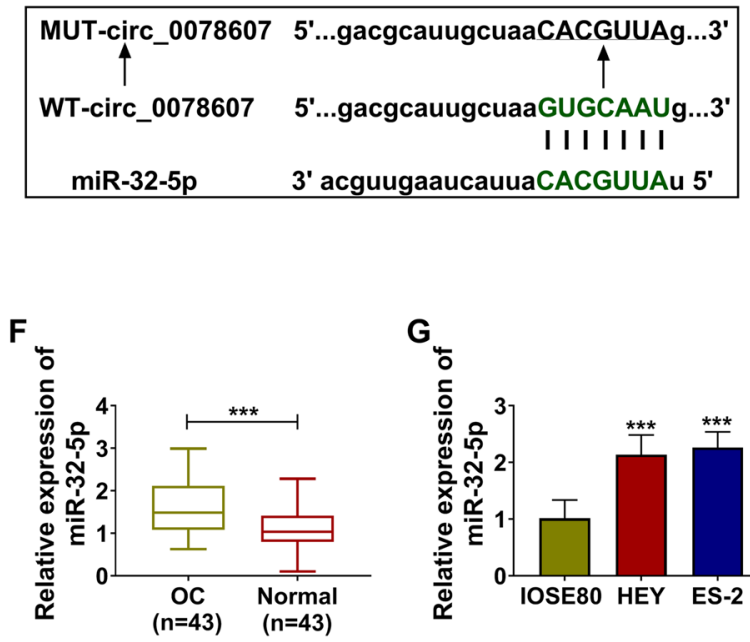

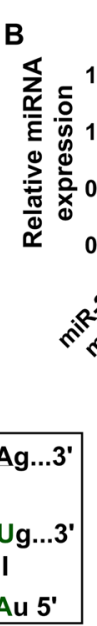

G.

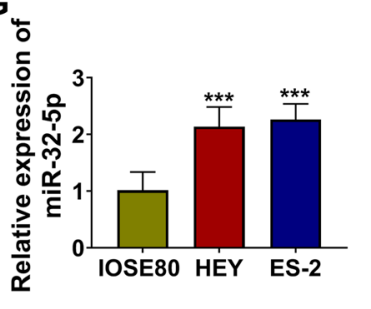

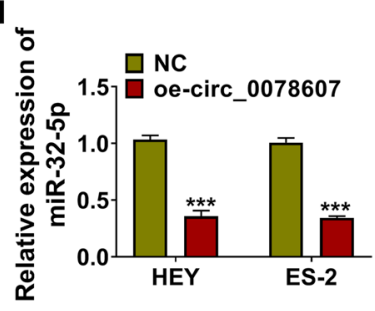
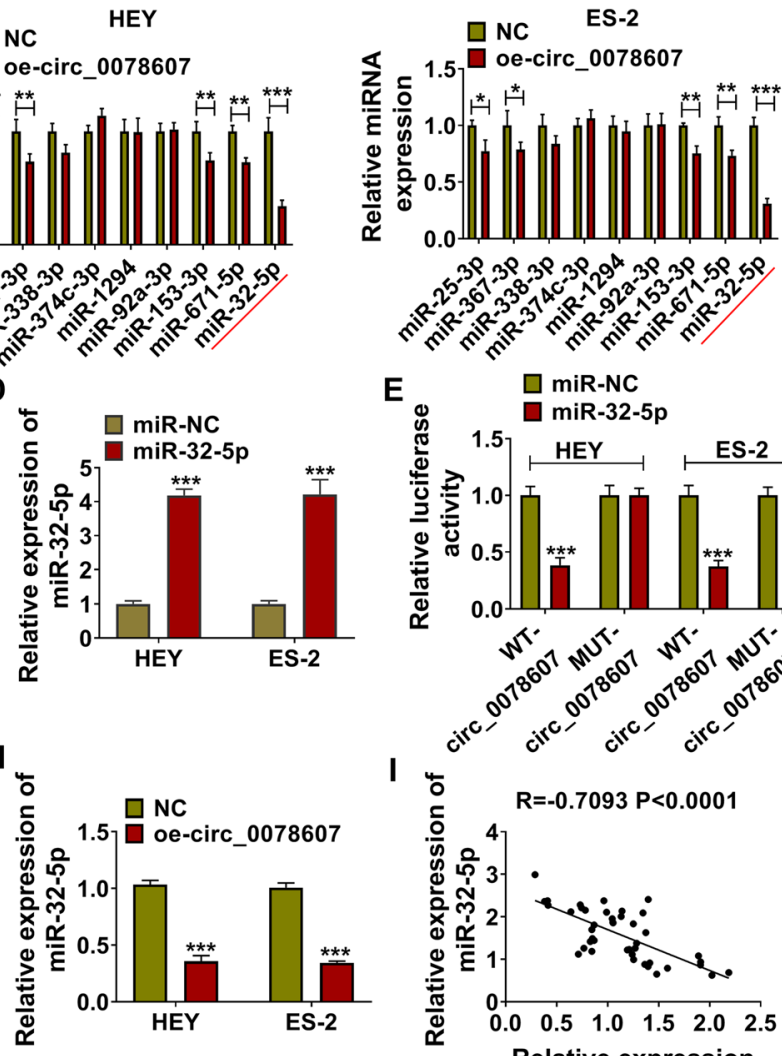

$\square$ miR-NC

$\square$ miR-32-5p
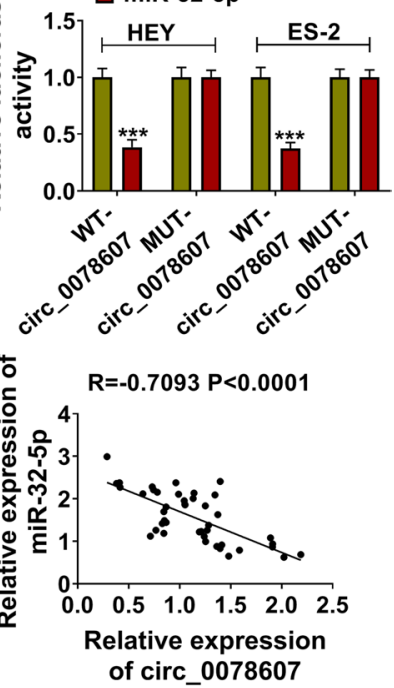

Fig. 5 Circ_0078607 could act as a sponge of miR-32-5p. A Venn diagram shows candidate miRNAs for circ_0078607. B The expression of candidate miRNAs was detected by qRT-PCR in HEY and ES-2 cells transfected with NC or oe-circ_0078607. C The binding sites between circ_0078607 and miR-32-5p were shown. D The transfection efficiency of miR-32-5p mimic in HEY and ES-2 cells was assessed by detecting miR-32-5p expression using qRT-PCR. E Dual-luciferase reporter assay was performed to verify the interaction between circ_0078607 and miR-32-5p. F QRT-PCR was used to measure the expression of miR-32-5p in OC tumor tissues (OC, $n=43$ ) and adjacent normal tissues (Normal, $n=43$ ). G MiR-32-5p expression in IOSE80 cells and both OC cells (HEY and ES-2) was tested by qRT-PCR. $\mathbf{H}$ The expression of miR-32-5p in HEY and ES-2 cells transfected with NC or oe-circ_0078607 was detected by qRT-PCR. I The correlation between circ_0078607 and miR-32-5p in OC tumor tissues ( $n=43$ ) was analyzed using Pearson correlation analysis. ${ }^{* *} P<0.001$

to be negatively correlated with miR-32-5p expression (Fig. 7F). Furthermore, we also uncovered that the promoting effect of circ_0078607 overexpression on the mRNA and protein expression levels of SIK1 could be reversed by miR-32-5p overexpression (Fig. 7G-H). Therefore, we confirmed that circ_0078607 sponged miR-32-5p to regulate SIK1.

The negative regulation of circ_0078607 on OC progression could be reversed by SIK 1 silencing

To further confirm that the regulation of circ_0078607 on OC progression was achieved by mediating SIK1 expression, oe-circ_0078607 and si-SIK1 were co-transfected into HEY and ES-2 cells. Through measuring the mRNA and protein expression levels of SIK1, we found that the enhancing effect of circ_0078607 on SIK1 expression could be inhibited by si-SIK1 (Fig. 8A-B). Further experiments revealed that knockdown of SIK1 could abolish the inhibitory effects of circ_0078607 overexpression on the EDU positive cells, the number of colonies and the numbers of migrated and invaded cells (Fig. 8C-F and Supplementary Fig. 2A-C). Besides, silenced SIK1 also reversed the increasing effect of circ_0078607 overexpression on the apoptosis rate of HEY and ES-2 cells (Fig. 8G and Supplementary Fig. 2D). The reducing effect of circ_0078607 on N-cadherin and MMP9 protein levels, as well as the promoting effect on E-cadherin and Bax protein levels also could be recovered by SIK1 knockdown in HEY and ES-2 cells (Fig. 8H). Our data showed that circ_0078607 regulated OC progression by regulating SIK1. 


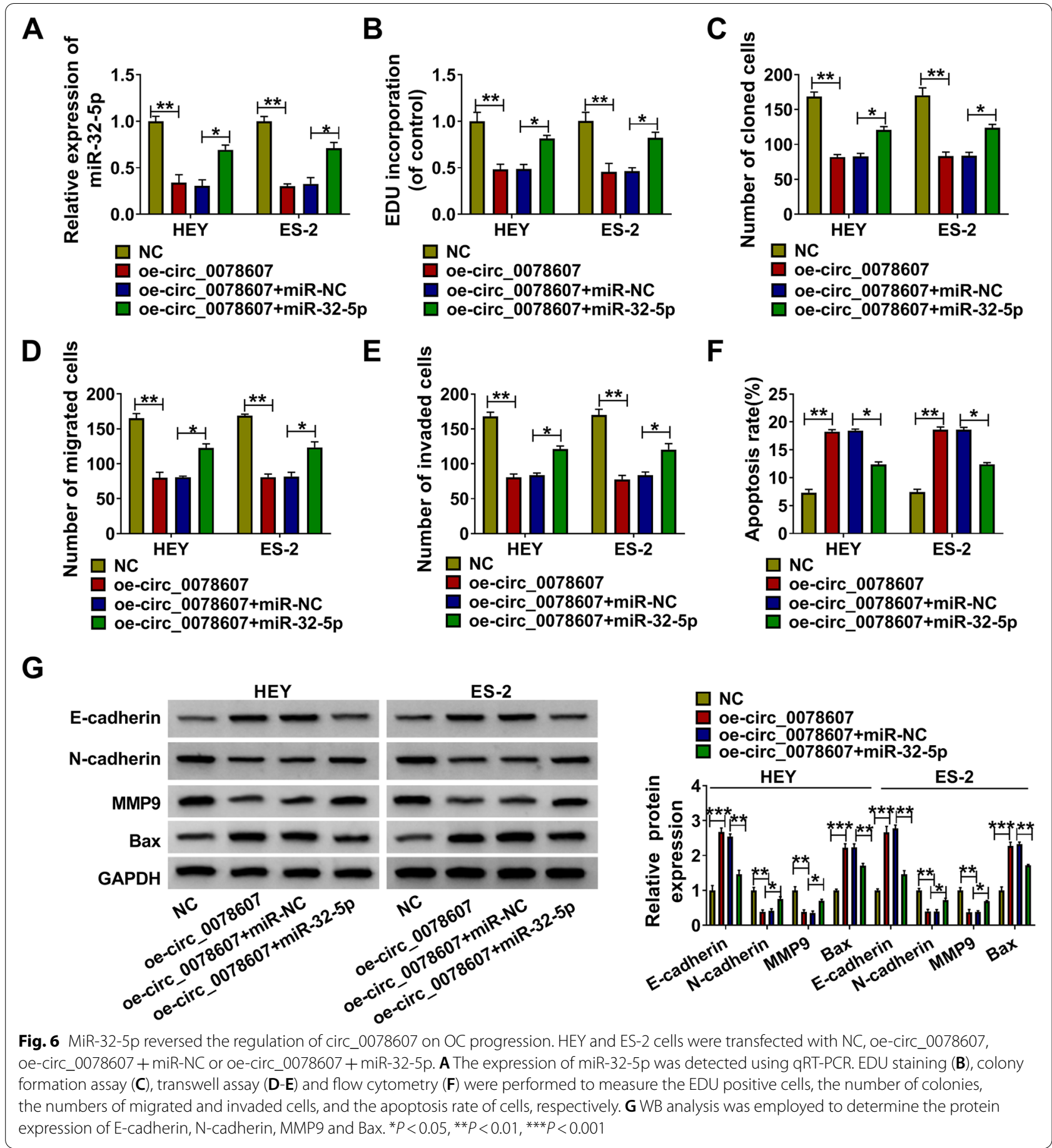

Circ_0078607 overexpression inhibited OC tumorigenesis For further investigating the role of circ_0078607 in OC, we assessed the effect of circ_0078607 overexpression on $\mathrm{OC}$ tumorigenesis in vivo. After 28 days of subcutaneous xenograft tumors construction, we found that tumor volume and weight in the circ_0078607 overexpression group were significantly lower than those in the control group (Fig. 9A-B). IHC staining results showed that the Ki-67 positive cells were markedly reduced in the tumor tissues of the lenti-circ_0078607 group (Fig. 9C). By detecting circ_0078607 expression in the tumors, we confirmed that circ_0078607 was indeed overexpressed in the circ_0078607 overexpression group (Fig. 9D). At the 


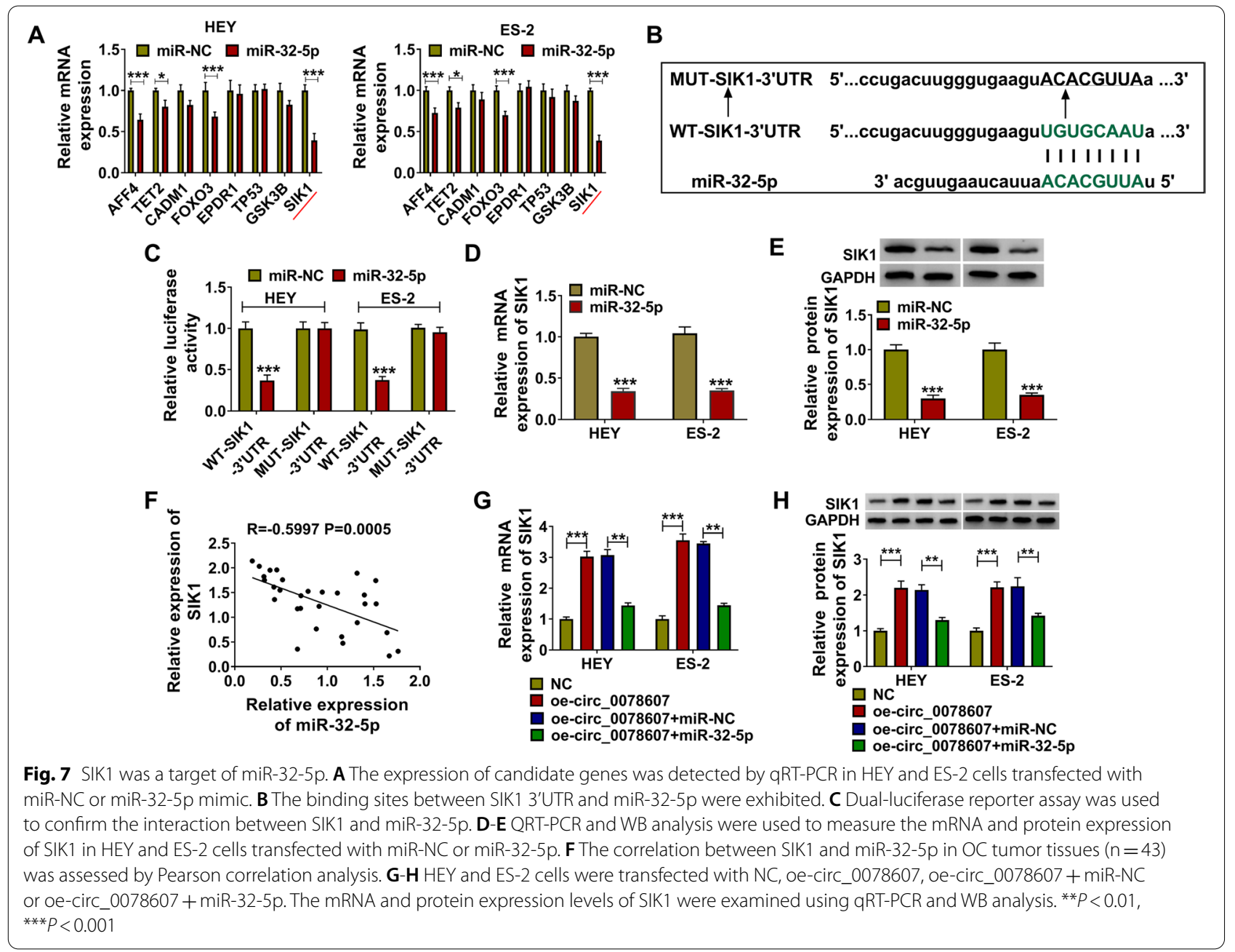

same time, we also found that miR-32-5p expression was obviously reduced, while SIK1 mRNA and protein expression levels were markedly enhanced in the circ_0078607 overexpression group (Fig. 9D-E). All data indicated that circ_0078607 suppressed OC tumorigenesis by regulating the miR-32-5p/SIK1 axis.

\section{Discussion}

In many studies, circRNA has been confirmed to be associated with cancer malignant progression, and different circRNA has different roles in different cancers. Li et al. showed that circTGFBR2 could hinder nasopharyngeal carcinoma proliferation and migration via sponging miR107 [10]. CircCUL2 was downregulated in gastric cancer and it could repress cancer malignant transformation and promote cisplatin sensitivity by sponging miR-142-3p and regulating ROCK2 [14]. In addition, circ_100146 had been discovered to be upregulated in bladder cancer, which could facilitate cell proliferation and metastasis via the miR-149-5p/RNF2 axis [21]. However, there are few studies on circ_0078607. Our study showed that circ_0078607 was underexpressed in OC tissues and cells, and it had an anti-proliferation, anti-metastasis, and pro-apoptosis function in OC. Animal experiments also indicated that circ_0078607 overexpression also could reduce $\mathrm{OC}$ tumorigenesis in vivo. Our data proposed that circ_0078607 play a negative role in OC progression, which was consistent with the reported results of Zhang et al. [29].

In previous studies, SIK1 has been found to have highly effective in preventing tumor formation and cancer progression $[6,25]$. In our study, we discovered a significant positive correlation between circ_0078607 and SIK1 with low expression in OC tumor tissues. SIK1 had been proved to be a tumor suppressor to regulate OC progression, which was similar to the effect of circ_0078607 on $\mathrm{OC}$ progression and consistent with the previous research results [3]. The analysis result of the functional test showed that SIK1 had an inhibitory effect on OC cell proliferation and metastasis, and could promote cell 


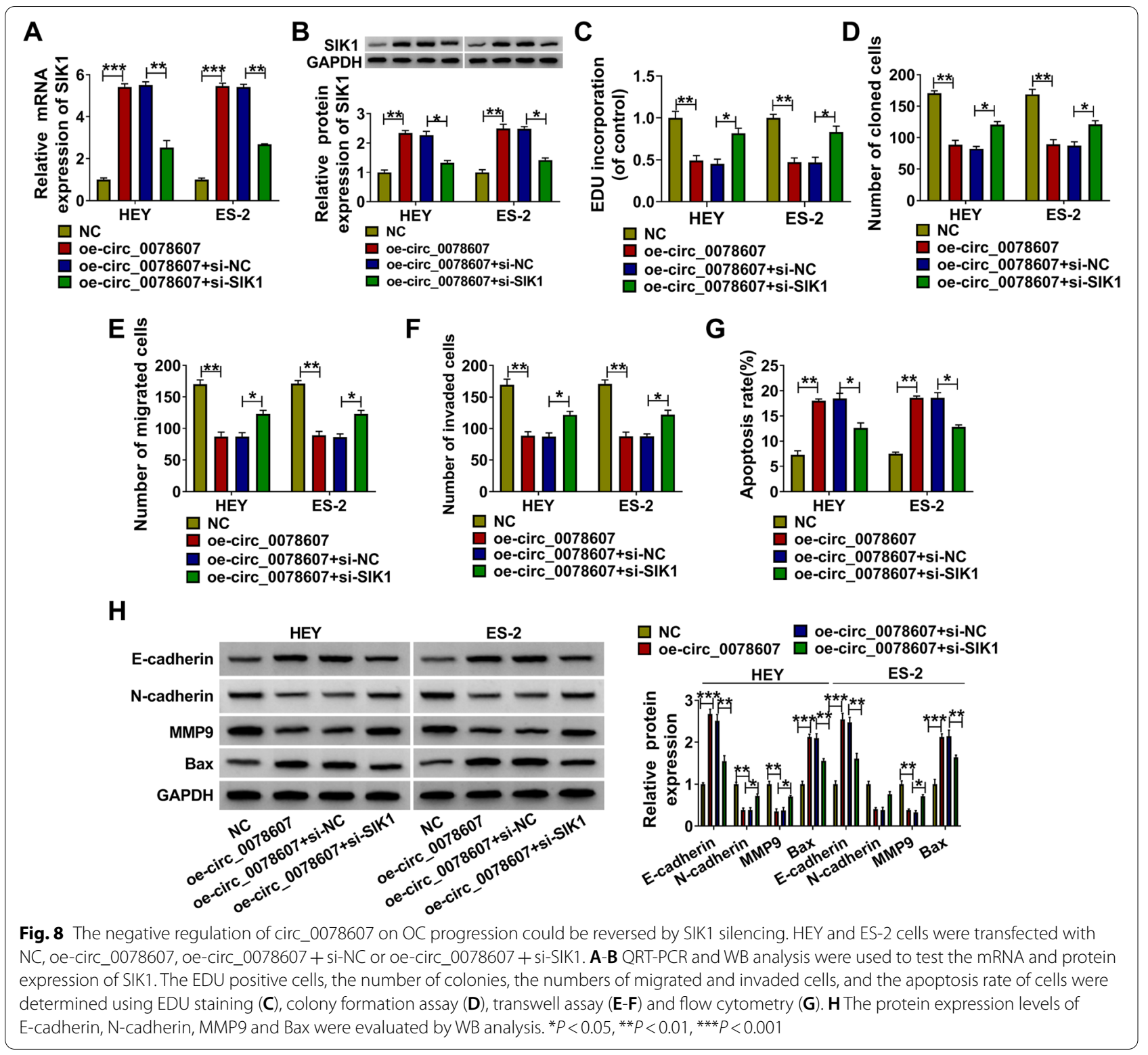

apoptosis. Considering that circRNA can indirectly regulate mRNA expression by sponging miRNA, we hypothesized that there was an interacted miRNA, which could be sponged by circ_0078607 and could target SIK1. After bioinformatics analysis and screening, we locked miR-32-5p.

MiR-32-5p has different functions in different cancers. Liang et al. showed that miR-32-5p had high expression in colorectal cancer, which could promote cancer cell metastasis and radioresistance [11]. In contrast, miR$32-5 p$ was discovered to restrain breast cancer proliferation and increase apoptosis [23]. In OC, miR-32-5p was found to enhance OC proliferation and metastasis by targeting SMG1 [27]. Consistent with this, our study also uncovered the high expression of miR-32-5p in OC tissues and cells. The reversal effect of miR-32-5p on circ_0078607 regulated OC progression suggested that circ_0078607 indeed sponged miR-32-5p to be involve in the regulation on $\mathrm{OC}$ proliferation, metastasis and apoptosis. As a target of miR-32-5p, SIK1 silencing also reversed the regulation of circ_0078607 on OC progression. In vivo experiments confirmed that circ_0078607 upregulated SIK1 to suppress OC tumorigenesis by sponging miR-32-5p. 

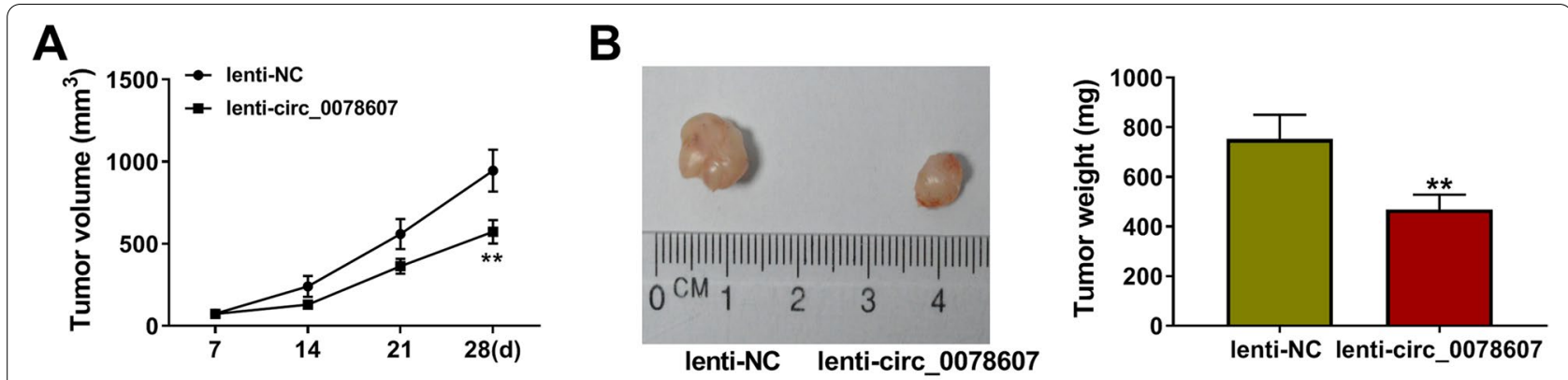

C

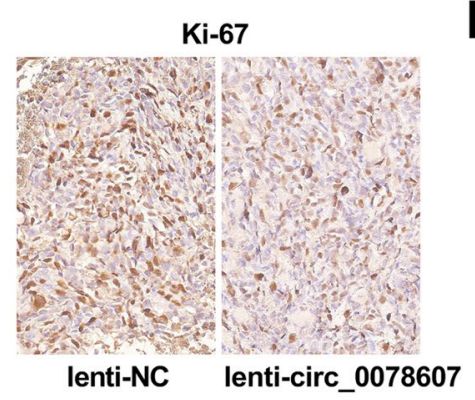

D

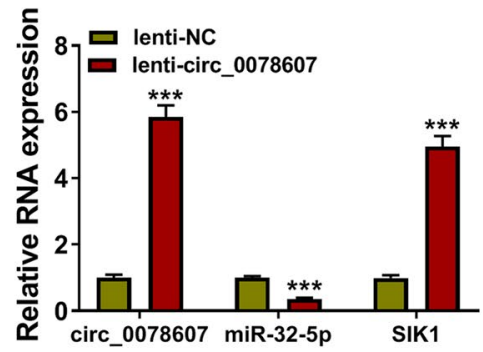

$\mathbf{E}$

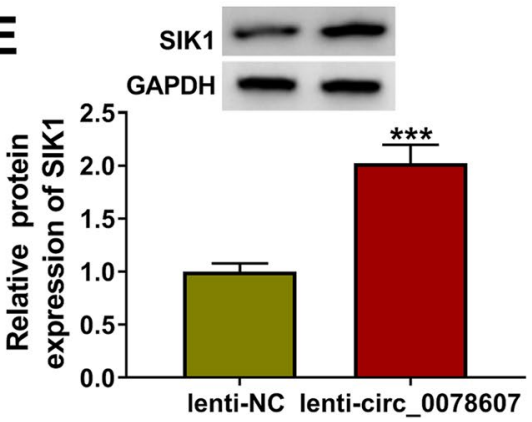

Fig. 9 Circ_0078607 overexpression inhibited OC tumorigenesis in vivo. HEY cells transfected with lenti-NC or lenti-circ_0078607 were injected into nude mice $(n=5)$. A Tumor volume was measured every 7 days until 28 days. B After 28 days, the tumors were removed and weighted. $\mathbf{C} I \mathrm{HC}$ staining was used to evaluate the Ki-67 positive cells in the tumor tissues. D The expression of circ_0078607, miR-32-5p and SIK1 in the tumors was detected using qRT-PCR. E The protein expression of SIK1 in the tumors was assessed using WB analysis. ${ }^{* *} P<0.01,{ }^{* * *} P<0.001$

In conclusion, our study revealed the role of circ_0078607 in OC progression and its potential new molecular mechanisms for regulating OC progression. Our data proposed that circ_0078607 restrained OC proliferation and metastasis through the miR-32-5p/ SIK1 axis. These results provided new evidence for circ_0078607 as a potential therapeutic target for OC.

\section{Supplementary Information}

The online version contains supplementary material available at https://doi. org/10.1186/s13048-021-00931-9.

Additional file 1: Supplementary Figure 1. The representative pictures for Fig. 6. The representative pictures for Fig. 6C (A), 6D (B), 6E (C) and 6F (D).

Additional file 2: Supplementary Figure 2. The representative pictures for Fig. 8. The representative pictures for Fig. 8C (A), 8D (B), 8E (C) and 8F (D).

\section{Acknowledgement}

Not applicable

\section{Authors' contributions}

Both authors read and approved the final manuscript.

\section{Funding}

No funding was received

\section{Availability of data and materials}

The analyzed data sets generated during the present study are available from the corresponding author on reasonable request.

\section{Declarations}

Ethics approval and consent to participate

The present study was approved by the ethical review committee of Liaocheng People's Hospital. Written informed consent was obtained from all enrolled patients.

\section{Consent for publication}

Patients agree to participate in this work.

\section{Competing interests}

The authors declare that they have no competing interests.

\section{Author details}

'Department of Reproductive Medicine, Liaocheng People's Hospital, NO.67, Dongchang West Road, Liaocheng City 252000, Shandong Province, China.

2Department of Obstetrics and Gynecology, Liaocheng Tird People's Hospital, Liaocheng, Shandong, China.

Received: 20 April 2021 Accepted: 1 December 2021

Published online: 04 January 2022

\section{References}

1. Barrett SP, Salzman J. Circular RNAs: analysis, expression and potential functions. Development. 2016;143(11):1838-47. https://doi.org/10.1242/ dev.128074. 
2. Belousova EA, Filipenko ML, Kushlinskii NE. Circular RNA: new regulatory molecules. Bull Exp Biol Med. 2018;164(6):803-15. https://doi.org/10. 1007/s10517-018-4084-z.

3. Chen JL, Chen F, Zhang TT, Liu NF. Suppression of SIK1 by miR141 in human ovarian cancer cell lines and tissues. Int J Mol Med. 2016;37(6):1601-10. https://doi.org/10.3892/ijmm.2016.2553.

4. Elsherif SB, Faria SC, Lall C, Iyer R, Bhosale PR. Ovarian Cancer genetics and implications for imaging and therapy. J Comput Assist Tomogr. 2019:43(6):835-45. https://doi.org/10.1097/RCT.0000000000000932.

5. Hansen $\mathrm{TB}$, Jensen $\mathrm{TI}$, Clausen $\mathrm{BH}$, et al. Natural RNA circles function as efficient microRNA sponges. Nature. 2013;495(7441):384-8. https://doi. org/10.1038/nature11993.

6. Hollstein PE, Eichner LJ, Brun SN, et al. The AMPK-related kinases SIK1 and SIK3 mediate key tumor-suppressive effects of LKB1 in NSCLC. Cancer Discov. 2019;9(11):1606-27. https://doi.org/10.1158/2159-8290.CD-18-1261.

7. Huang C, Liu J, Xu L, et al. MicroRNA-17 promotes cell proliferation and migration in human colorectal cancer by downregulating SIK1. Cancer Manag Res. 2019:11(3521-34. https://doi.org/10.2147/CMAR.S191087.

8. Kim A, Ueda Y, Naka T, Enomoto T. Therapeutic strategies in epithelial ovarian cancer. J Exp Clin Cancer Res. 2012;31(14. https://doi.org/10. 1186/1756-9966-31-14.

9. Kossai M, Leary A, Scoazec JY, Genestie C. Ovarian Cancer: a heterogeneous disease. Pathobiology. 2018;85(1-2):41-9. https://doi.org/10.1159/ 000479006

10. Li W, Lu H, Wang H, et al. Circular RNA TGFBR2 acts as a ceRNA to suppress nasopharyngeal carcinoma progression by sponging miR-107. Cancer Lett. 2020. https://doi.org/10.1016/j.canlet.2020.11.001.

11. Liang $H$, Tang $Y$, Zhang H, Zhang C. MiR-32-5p regulates Radiosensitization, migration and invasion of colorectal Cancer cells by targeting TOB1 gene. Onco Targets Ther. 2019;12:9651-61. https://doi.org/10.2147/OTT. S228995.

12. Narod S. Can advanced-stage ovarian cancer be cured? Nat Rev Clin Oncol. 2016;13(4):255-61. https://doi.org/10.1038/nrclinonc.2015.224.

13. Panda AC. Circular RNAs act as miRNA sponges. Adv Exp Med Biol. 2018;1087:67-79. https://doi.org/10.1007/978-981-13-1426-1_6.

14. Peng $L$, Sang $H$, Wei $S$, et al. circCUL2 regulates gastric cancer malignant transformation and cisplatin resistance by modulating autophagy activation via miR-142-3p/ROCK2. Mol Cancer. 2020;19(1):156. https://doi.org/ 10.1186/s12943-020-01270-X.

15. Qu, C., He, Lu, X., et al. Salt-inducible kinase (SIK1) regulates HCC progression and WNT/beta-catenin activation. J Hepatol 2016; 64(5):1076-89. https://doi.org/10.1016/j.jhep.2016.01.005.

16. Ren ZG, Dong SX, Han P, Qi J. miR-203 promotes proliferation, migration and invasion by degrading SIK1 in pancreatic cancer. Oncol Rep. 2016;35(3):1365-74. https://doi.org/10.3892/or.2015.4534.

17. Rooth C. Ovarian cancer: risk factors, treatment and management. Br J Nurs. 2013;22(17):S23-30. https://doi.org/10.12968/bjon.2013.22.Sup17. S23.

18. Sakamoto K, Bultot L, Goransson O. The salt-inducible kinases: emerging metabolic regulators. Trends Endocrinol Metab. 2018;29(12):827-40. https://doi.org/10.1016/j.tem.2018.09.007.

19. Shabaninejad Z, Vafadar A, Movahedpour A, et al. Circular RNAs in cancer: new insights into functions and implications in ovarian cancer. J Ovarian Res. 2019;12(1):84. https://doi.org/10.1186/s13048-019-0558-5.

20. Stewart C, Ralyea C, Lockwood S. Ovarian Cancer: an integrated review. Semin Oncol Nurs. 2019;35(2):151-6. https://doi.org/10.1016/j.soncn. 2019.02.001.

21. Wang H, Niu X, Mao F, et al. Hsa_circRNA_100146 acts as a sponge of miR-149-5 $p$ in promoting bladder Cancer progression via regulating RNF2. Onco Targets Ther. 2020;13:11007-17. https://doi.org/10.2147/OTT. S273622.

22. Wang J, Wu A, Yang B, et al. Profiling and bioinformatics analyses reveal differential circular RNA expression in ovarian cancer. Gene. 2020;724:144150. https://doi.org/10.1016/j.gene.2019.144150.

23. Wang R, Huang Z, Qian C, et al. LncRNA WEE2-AS1 promotes proliferation and inhibits apoptosis in triple negative breast cancer cells via regulating miR-32-5p/TOB1 axis. Biochem Biophys Res Commun. 2020;526(4):100512. https://doi.org/10.1016/j.bbrc.2020.01.170.

24. Wein MN, Foretz M, Fisher DE, Xavier RJ, Kronenberg HM. Salt-inducible kinases: physiology, regulation by CAMP, and therapeutic potential.
Trends Endocrinol Metab. 2018;29(10):723-35. https://doi.org/10.1016/j. tem.2018.08.004

25. Yang L, Xie N, Huang J, et al. SIK1-LNC represses the proliferative, migrative, and invasive abilities of lung cancer cells. Onco Targets Ther. 2018;11:4197-206. https://doi.org/10.2147/OTT.S165278.

26. Yong Kim S, Jeong S, Chah KH, et al. Salt-inducible kinases 1 and 3 negatively regulate toll-like receptor 4-mediated signal. Mol Endocrinol. 2013;27(11):1958-68. https://doi.org/10.1210/me.2013-1240.

27. Zeng S, Liu S, Feng J, Gao J, Xue F. MicroRNA-32 promotes ovarian cancer cell proliferation and motility by targeting SMG1. Oncol Lett. 2020;20(1):733-41. https://doi.org/10.3892/ol.2020.11624.

28. Zhang L, Zhou Q, Qiu Q, et al. CircPLEKHM3 acts as a tumor suppressor through regulation of the miR-9/BRCA1/DNAJB6/KLF4/AKT1 axis in ovarian cancer. Mol Cancer. 2019;18(1):144. https://doi.org/10.1186/ s12943-019-1080-5.

29. Zhang N, Jin Y, Hu Q, et al. Circular RNA hsa_circ_0078607 suppresses ovarian cancer progression by regulating miR-518a-5p/Fas signaling pathway. J Ovarian Res. 2020;13(1):64. https://doi.org/10.1186/ s13048-020-00664-1.

30. Zong ZH, Du YP, Guan X, Chen S, Zhao Y. CircWHSC1 promotes ovarian cancer progression by regulating MUC1 and hTERT through sponging miR-145 and miR-1182. J Exp Clin Cancer Res. 2019;38(1):437. https://doi. org/10.1186/s13046-019-1437-z.

\section{Publisher's Note}

Springer Nature remains neutral with regard to jurisdictional claims in published maps and institutional affiliations.

Ready to submit your research? Choose BMC and benefit from:

- fast, convenient online submission

- thorough peer review by experienced researchers in your field

- rapid publication on acceptance

- support for research data, including large and complex data types

- gold Open Access which fosters wider collaboration and increased citations

- maximum visibility for your research: over 100M website views per year

At BMC, research is always in progress.

Learn more biomedcentral.com/submissions 